\title{
Clonidine was effective for reducing tamoxifen associated hot flashes in postmenopausal women with breast cancer
}

\author{
Pandya KJ, Raubertas RF, Flynn PJ, et al. Oral clonidine in postmenopausal patients with breast cancer experiencing \\ tamoxifen-induced hot flashes: a University of Rochester Cancer Center Community Clinical Oncology Program Study. \\ Ann Intern Med 2000 May 16;132:788-93. \\ QUESTION: In postmenopausal women receiving tamoxifen for breast cancer, does \\ clonidine reduce hot flashes?
}

\section{Design}

Randomised \{allocation concealed*\} $\uparrow$, blinded \{clinicians, patients, and outcome assessors $\}+, *$ placebo controlled trial with 12 weeks follow up.

\section{Setting}

University of Rochester Cancer Center Community Clinical Oncology Program, New York, United States.

\section{Patients}

198 postmenopausal women who had received adjuvant tamoxifen treatment for breast cancer for $\geqslant 1$ month and who reported $\geqslant 1$ hot flash per day. Exclusion criteria were concurrent chemotherapy or other endocrine treatment for breast cancer; use of antihypertensive drugs, monoamine oxidase inhibitors, L-dopa, piribedil, tricyclic antidepressants, or sedatives; coronary insufficiency; myocardial infarction in the previous 3 months; symptomatic cardiac disease; peripheral or cerebrovascular disease; syncope; symptomatic hypertension; inability to tolerate clonidine; or abnormal renal or hepatic function. 194 women provided baseline data (mean age 54 y). Follow up was $91 \%$ at week $4,82 \%$ at week 8 , and $75 \%$ at week 12 .

\section{Intervention}

After stratification for time since menopause, duration of tamoxifen treatment, and baseline frequency of hot flashes, patients were allocated to oral clonidine, 0.1 $\mathrm{mg} /$ day $(\mathrm{n}=99)$, or placebo $(\mathrm{n}=99)$ for 8 weeks.

\section{Main outcome measures}

Hot flash symptoms (frequency; mean severity grade [score range $1=$ mild to $4=$ very severe]; mean duration; and combined score for frequency, severity, and duration); quality of life (self report scale with scores ranging from $1=$ worst possible life to $10=$ best possible life); and side effects.

\section{Main results}

The clonidine group reported a greater reduction in the frequency of hot flashes at 4 weeks (mean percentage reduction $37 \%$ v 20\%, 95\% CI for the $17 \%$ difference $7 \%$

Source of funding:

National Cancer

Institute.

For correspondence: Dr KJ Pandya,

University of Rochester

Cancer Center, 601

Elmwood Avenue, Box

704, Rochester, $N Y$

14642, USA. Fax +1

7162731051 to $27 \%$ ) and 8 weeks (mean percentage reduction $38 \% v$ $24 \%$, CI for the $14 \%$ difference $3 \%$ to $27 \%$ ). The reduction in the hot flash score from baseline was greater in the clonidine group than in the placebo group at 4 weeks (mean difference in percentage reduction $18.5 \%$, $\mathrm{p}=0.002$ ) and 8 weeks (mean difference in percentage reduction $18.6 \%, \mathrm{p}=0.006$ ). Differences between groups for hot flash severity and duration were not statistically significant at 4 and 8 weeks. The clonidine group had greater improvements from baseline in quality of life

scores than did the placebo group at 4 weeks (mean score change from baseline $0.4 v-0.3, \mathrm{p}=0.003$ ) and 8 weeks (mean score change from baseline $0.3 v-0.2$, $\mathrm{p}=0.022)$. More women in the clonidine group than in the placebo group reported difficulty in sleeping $(41 \% v$ $21 \%, \mathrm{p}=0.02$ ).

\section{Conclusion}

In postmenopausal women who were receiving tamoxifen for breast cancer, clonidine was effective for reducing hot flashes.

*See glossary.

†Information provided by author.

\section{COMMENTARY}

The results of the study by Pandya et al can be compared with those of another similarly conducted trial by the North Central Cancer Treatment Group (NCCTG). ${ }^{1}$ This latter trial was a randomised, double blind, placebo controlled crossover trial $(n=116)$ of a transdermal clonidine preparation (1 transdermal therapeutic system).

These 2 trials had remarkably similar results. In the NCCTG trial, the intervention group had a greater reduction in hot flash score than did the control group (mean reduction in hot flash score $56 \% v 30 \%, \mathrm{p}<0.04$ ). In these trials, placebos were associated with hot flash score reductions of $24 \%$ and $30 \%$, whereas clonidine was associated with hot flash score reductions of $42 \%$ and $56 \%$.

Both trials noted substantially more toxicity in the patients receiving clonidine. In the study by Pandya et al, clonidine was associated with greater sleeping difficulty. In the NCCTG trial, the clonidine group had substantially more trouble with mouth dryness, constipation, drowsiness, and itchiness under the transdermal patch.

Pandya et al noted a statistically significant improvement in quality of life in the clonidine group. The reported 4\% increase in quality of life in the clonidine group compared with a $3 \%$ decrease in the placebo group, however, is quite modest. In the NCCTG trial, a specific quality of life question was not addressed, although patients were asked which of the 2 treatment periods they preferred after the double blind study periods were completed. The response to this query did not show a substantial preference for the clonidine group, presumably because of toxicity considerations.

Clonidine is an option for treating hot flashes in women with breast cancer but is limited by the toxicity associated with it.

Charles L Loprinzi, MD Mayo Clinic

Rochester, Minnesota, USA

1 Goldberg RM, Loprinzi CL, O'Fallon JR, et al. Transdermal clonidine for ameliorating tamoxifen-induced hot flashes. $J$ Clin Oncol 1994;12:155-8. 